\title{
On the Rectification of Vibratory Motion
}

\author{
R. W. BROCKETT \\ Division of Applied Sciences, Harvard University, Cambridge, MA 02138 (U.S.A.)
}

\begin{abstract}
This paper establishes a theoretical framework for analyzing and classifying actuators that generate their output by rectifying small-amplitude mechanical vibrations, such as might be generated by piezoelectric elements. These ideas are of special interest when designing for microfabrication, because motors based on these principles: (a) can generate translational output directly without use of rotary bearings; (b) appear to be scalable over several orders of magnitude of the length scale; and (c) appear to be capable of generating mechanical power proportional to driving frequency over one to two orders of magnitude of frequency. In order to achieve this performance, it is necessary to be able to fabricate features, or at least control surface irregularities, on the scale $v / w$ where $v$ is velocity of the actuator and $w$ is the operating frequency.
\end{abstract}

\section{Introduction}

Over the past few years there have been a number of papers written about the design of small electric motors. For the most part, the designs considered have been based on the use of electrostatic forces [1-3]. Such systems are particularly attractive if high speed, low torque, rotary motion is desired. Although intended for largerscale applications, recently several interesting designs based on other principles have also appeared, such as standing wave ('inchworm') designs [4] and the traveling wave motor [5]. In this paper we consider generic designs of high force, low velocity, motors, with the study of linear motion generation being of special interest.

Our point of view is derived from the following observations:

(a) A variety of materials are available which convert electrical energy to mechanical energy through the piezoelectric effect. These are low strain, high force effects.

(b) Such materials can effectively generate low-amplitude mechanical vibrations in the 200 $200000 \mathrm{~Hz}$ range, with higher frequencies yielding greater power per unit volume. (c) In order to generate large-amplitude displacements using this transduction mechanism, it is necessary to 'rectify' the mechanical oscillation.

We will refer to devices that transform reciprocating mechanical motion into unidirectional, rectilinear or rotary motion as mechanical rectifiers.

It may be useful to draw attention to an analogy with microwave engineering. In the 1930s and 1940s efforts directed toward solving problems arising in connection with generating and transmitting high-frequency power for radar, communication, and eventually microwave heating, changed the subject of electrical engineering in a profound way. Key developments included the coupled wave analysis of traveling wave tubes, parametric amplifiers, the frequency power (Manley-Rowe) equations and the steady development of ever better materials. Of course, high-frequency mechanical vibrations in the ultrasonic range are already widely used in acoustical imaging, nondestructive testing and in a variety of niche markets. Recently several different types of piezoelectric motors have become available, and consumer products based on them have been announced. Surface acoustic wave devices and optoacoustic elements have already found a place in electronics. Clearly this is a time of rapidly expanding possibilities for ultrasonic engineering, paralleling at least vaguely the earlier growth of microwave engineering.

It has been suggested in the literature that certain aspects of mechanics need to be rethought insofar as they are to be applied to high-frequency vibratory systems. In particular, static friction seems to be reduced when the contacting surfaces are subjected to a small-amplitude, high-frequency oscillation. The attention of the reader is directed to the survey [9] for a discussion of a number of interesting questions related to these and other matters.

\section{The Classical Situation}

The slider/crankshaft mechanism is, of course, the usual model for mechanical rectification. It converts reciprocating motion to rotational

Elsevier Sequoia/Printed in The Netherlands 
motion and can, with the addition of a rack and pinion, convert reciprocating motion into rectilinear motion. There are two reasons why this mechanism does not seem to be particularly well suited for microfabrication. In the first place, the rotational velocity of the crankshaft equals the frequency of the reciprocating motion; highfrequency vibration equates to high rotational speeds. A second problem is that this coupling produces no mechanical advantage. If mechanical advantage is required, it must be generated by a separate mechanism such as gearing. It is also worth noting that the offset of the crankshaft equals one-half the amplitude of the reciprocating motion. Thus for piezoelectric systems, the offset of the crankshaft would appear to be unacceptably small and the rotational velocities unacceptably large.

In view of this, it is not surprising that the commercially available piezoelectric motors do not use slider/crankshaft kinematics, but instead use a variety of more subtle methods. In this paper we suggest a classification for a range of possibilities making use of a geometric viewpoint. Our goal is to establish the framework for a general theory and to illustrate its applicability with examples.

\section{Differential Equation Models}

From the point of view of classical mechanics, rectifiers are necessarily non-holonomic systems. This remark is to be understood in the following sense. When the vibrating transducer completes one cycle, returning to its original position, the output element to which it is coupled must not return to its initial position. If it were to do so, no rectification could have occurred. Instead after one cycle, the output element will be offset by a certain amount; this is the essence of the function of the rectifier. Thus although the vibrator and the output must be coupled, there can be no finite set of smooth functions $\phi\left(x_{1}, x_{2}, \ldots, x_{n}\right)$ such that the only constraints relating the position of the vibrator and the output are of the form $\phi\left(x_{1}, x_{2}, \ldots, x_{n}\right)=0$. It will typically happen that the relation between the position of the vibrator and the output will take the form of one or more relationships between the velocities of the separate pieces, these constraints being of such a form that they cannot be integrated to give position constraints. Standard textbooks on dynamics usually cite the example of a disk or a ball rolling on a surface without slipping. See, e.g., Whittaker [6]. In addition, there is a second type of non-holonomic situation that arises. This occurs in systems for which there are certain regimes during which parts are in contact and move together constrained by structural features or friction, and other regimes during which they are separate and go their own ways. A formalism for studying such 'piecewise holonomic' systems has been developed [10].

In this paper we will discuss a number of mechanisms that realize rectification. We organize the discussion along the lines suggested by Fig. 1 . The distinctions drawn there are based on the number of driving vibrators (this is analogous to the classification of electric motors as single phase or multiple phase) and on the type of coupling (distinguishing between smooth non-holonomic systems and piecewise holonomic systems). In all cases we will be able to model the input/output relations by a set of differential equations.

The remainder of this Section is devoted to the discussion of some aspects of ordinary differential equations that are important for the results on vibratory actuators which follow.

Of course, there is no theory of periodic solutions of non-linear differential equations that is both quantitative and completely general. However, there is an effective theory based on the assumption that the non-linear part of the differential equation is small and certain aspects of this theory are useful here. Starting with a vector equation

$$
\dot{x}(t)=f(x(t), t)
$$

with $f(x, \cdot)$ being periodic, one introduces a change of coordinates of the form $z(t)=P(t) x(t)$ with $P$ being periodic. This gives

$$
\dot{z}(t)=\dot{P}(t) P^{-1}(t) z(t)+P(t) f\left(P^{-1}(t) z(t), t\right)
$$

It is then postulated that $P$ has been selected so that this can be rewritten as

$\dot{z}(t)=\epsilon g(z(t), t)$

with $\epsilon$ a small parameter, $g(0, t)=0$ and $g(z, \cdot)$ periodic with respect to $t$. This being the case, we suppose that $g(z, \cdot)$ is periodic with period $T$ and look for a solution $z(\cdot)$, which is the sum of a constant, a periodic term and a linear term, with

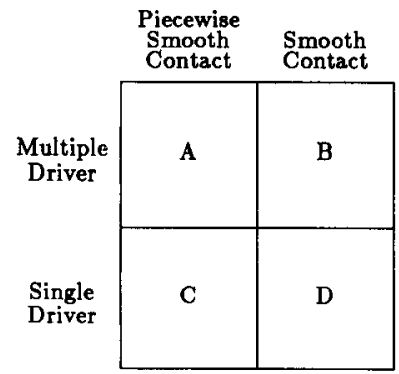

Fig. 1. A mechanical rectifier taxonomy. 
weights $\epsilon^{0}, \epsilon^{1}, \epsilon^{2}$, respectively. That is, we search for a solution of the form

$z(t)=z_{0}+\epsilon p(t)+\epsilon^{2} \alpha t$

with $z_{0}$ and $\alpha$ being constant vectors and $p(t)$ being periodic with period $T$. The $\epsilon^{2} \alpha t$ term is often said to be a secular term. In the situation of interest here, the vector $z$ represents the state of the entire system (e.g., the positions and velocities of all elements if we are discussing a lumped mechanical system). Since we want to convert periodic motion into rectilinear motion, we see that the secular term is of critical importance.

The analysis of $\dot{z}(t)=\epsilon g(z(t), t)$ for $\epsilon$ small proceeds by integrating both sides and using a Taylor series expansion. This yields

$\left.z(t)\right|_{0} ^{T}=\epsilon \int_{0}^{t} g\left(z_{0}+\epsilon p(\sigma)+\epsilon^{2} \alpha \sigma, \sigma\right) \mathrm{d} \sigma$

so that

$\epsilon^{2} \alpha T \approx \epsilon \int_{0}^{t} g\left(z_{0}, \sigma\right) \mathrm{d} \sigma+\epsilon \int_{0}^{t} \frac{\partial g}{\partial z}\left(\epsilon p(\sigma)+\epsilon^{2} \alpha \sigma\right) \mathrm{d} \sigma$

We define $G\left(z_{0}\right)$ as

$G\left(z_{0}\right)=\int_{0}^{t} g\left(z_{0}, \sigma\right) \mathrm{d} \sigma$

If $G\left(z_{0}\right)=0$ and if the constant $n$ by $n$ matrix obtained by evaluating $(\partial G / \partial z)$ at $z=z_{0}$ is of full rank, then there is a solution with $\alpha=0$ and this solution is unique in the sense that there is no other solution for small $\epsilon$. That is not, for our present purposes, the interesting case, because if $\partial G / \partial z$ is of full rank, there is no secular term. If $(\partial G / \partial z)$ is not of full rank, then there exists $\alpha$ such that $(\partial G / \partial z) \alpha=0$. If

$\epsilon^{2} \alpha T=\epsilon^{2} \int_{0}^{T}(\partial g / \partial z) p(\sigma) \mathrm{d} \sigma$

then, at least to first order in $t, z(t)=z_{0}+\epsilon p(t)+$ $\epsilon^{2} \alpha t$ is a possible solution. This kind of thinking can be worked into a rigorous theory (involving some additional assumptions, see Chapter 18 of Hale [7]) and we will use it to guide our later developments.

\section{The Role of Area}

Since the vector $\alpha$ represents the velocity of the rectified output, we are interested in determining how $\alpha$ depends on the properties of the system.
The following remarks will be useful in this regard.

Of course the area between the $x$-axis and the graph of the function $y=f(x)$ can be expressed as

$A(t)=\int_{0}^{t} y \dot{x} \mathrm{~d} \sigma$

provided that $x$ itself is a function of a monotonically increasing variable, say time. The area can also be expressed by integrating by parts:

$A(t)=\left.x(\sigma) y(\sigma)\right|_{0} ^{t}-\int_{0}^{t} \dot{y} x \mathrm{~d} \sigma$

If $x(t+T)=x(t)$ and $y(t+T)=y(t)$, then the curve $(x(\cdot), y(\cdot))$ is closed and the signed area encompassed by the curve is

$A=\frac{1}{2} \int_{0}^{t} x \dot{y}-y \dot{x} \mathrm{~d} t$

We want to state this in a different, but completely equivalent, way. If we have an input/ output system described by the equations

$\dot{x}(t)=u(t)$

$\dot{y}(t)=v(t)$

$\dot{z}(t)=x(t) v(t)-y(t) u(t)$

and if $u(\cdot)$ and $v(\cdot)$ are periodic functions with zero average value, then $x(\cdot)$ and $y(\cdot)$ are periodic and

$z(t)=\frac{2 A}{T} \cdot t+p(t)$

where $A=$ the area enclosed by the closed curve $(x(\cdot), y(\cdot))$ for $0 \leqslant t \leqslant T$ and $p(t)$ is a periodic function. Arguments given in our earlier paper [8] show that this control system is a canonical form for a two-input controllable system with no drift term. We now describe a sense in which it can be thought of as a canonical form for smooth vibrational rectification.

If we have two vibrators driving a dynamical system and include in our model dynamic effects as well as kinematic effects, the equation of motion will typically take the form

$\dot{x}=f(x)+g_{1}(x) u+g_{2}(x) v$

with $u$ and $v$ being the displacements of the vibratory elements. On the other hand, if only the kinematics are being modeled, then $f$ can be taken to be zero and the equation will take the form $\left(x_{\mathrm{r}}\right.$ is a reduced version of $x$ obtained by omitting the components which represent velocities)

$\dot{x}_{\mathrm{r}}=\tilde{g}_{1}\left(x_{\mathrm{r}}\right) u+\tilde{g}_{2}\left(x_{\mathrm{r}}\right) v$ 
As a control system, $u$ can drive $x_{\mathrm{r}}$ in the direction defined by the vector $\tilde{g}_{1}$ and $v$ can drive $x_{\mathrm{r}}$ in the direction defined by the vector $\tilde{g}_{2}$ but, and this is one of the things that makes non-linear control theory interesting, by working together they can also drive $x$ in the direction defined by the vector

$\left[\tilde{g}_{1}, \tilde{g}_{2}\right]=\frac{\partial \tilde{g}_{1}}{\partial x} \tilde{g}_{2}-\frac{\partial \tilde{g}_{2}}{\partial x} \tilde{g}_{1}$

If we choose coordinates so that $x_{\mathrm{r}}=0$ is the nominal operating point, and so that

$\tilde{g}_{1}(0)=\left[\begin{array}{c}1 \\ 0 \\ 0 \\ \vdots\end{array}\right] ; \quad \tilde{g}_{2}(0)=\left[\begin{array}{c}0 \\ 1 \\ 0 \\ \vdots\end{array}\right]$

then we can see that for $u$ and $v$ periodic of period $T$ and $x(0)=0$,

$x_{\mathrm{r}}(T) \approx\left[\tilde{g}_{1}, \tilde{g}_{2}\right] \cdot A$

with the vector $\left[\tilde{g}_{1}, \tilde{g}_{2}\right]$ being evaluated at $x_{\mathrm{r}}=0$ and $A$ being the area defined by the closed curve described by the first and second components of $x_{\mathbf{r}}$. Thus in an average sense

$\dot{x}_{\mathrm{r}} \approx\left[\tilde{g}_{1}, \tilde{g}_{2}\right] \cdot A / T$

\section{Type A Kinematic Realization}

We wish to describe prototype mechanisms for producing rectilinear motion by rectification using piecewise smooth kinematics. The basic mechanism is only slightly different from the completely smooth version to be discussed in the next Section.

Consider a beam supported at each end and which is being driven in such a way as to excite the first and second harmonics (see Figs. 2 and 3). If the beam is shaped so that these modes can be effectively excited at the same frequency and with arbitrary phase, then the shape takes the form

$y(t, w)=A \phi(w) \sin \omega t+B \psi(w) \sin (\omega t+\theta)$

Suppose that at point $w_{0}$ along the beam we add a small rectangular structure extending perpendicularly from the beam as shown. Since the

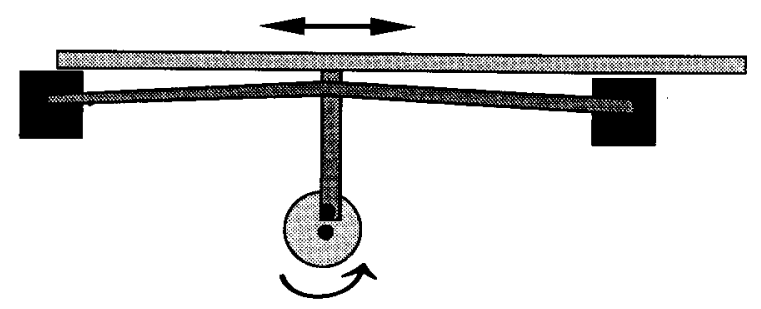

Fig. 2. Modes for the generation of linear motion.

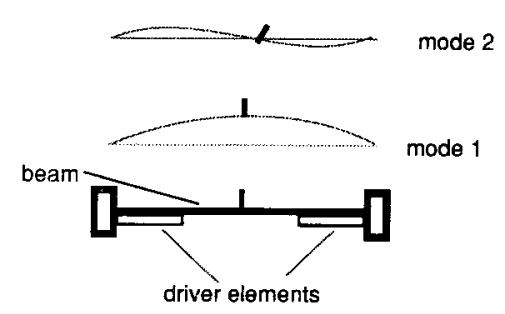

Fig. 3. Beam with normal extension.

extension is normal to the beam and since the vertical component of the unit normal to the beam at $w$ is (subscript denotes derivative)

$$
\begin{aligned}
& n_{y}(t, w)= \\
& \frac{A \phi_{w}(w) \sin \omega t+B \psi_{w}(w) \sin (\omega t+\theta)}{\left[1+\left(A \phi_{w}\left(w_{0}\right) \sin \omega t+B \phi_{w}(w) \sin (\omega t+\theta)\right)^{2}\right]^{1 / 2}}
\end{aligned}
$$

we see that the $x(t)$ and $y(t)$ displacements of the tip of the extension are, for small values of $A$ and $B$, approximately

$$
\begin{aligned}
& y\left(t, w_{0}\right)=h+A \phi_{w}(w) \sin \omega t+B \psi(w) \sin (\omega t+\theta) \\
& x\left(t, w_{0}\right)=h A \phi(w) \sin \omega t+h B \phi_{w}(w) \sin (\omega t+\theta)
\end{aligned}
$$

Thus the top of the extension moves in an ellipse. The principal axes of the ellipse are lined up with the vertical and horizontal axes and the area is proportional to $A B$.

\section{Type B Kinematic Realization}

In a type $A$ realization the governing equations are only piecewise smooth, because during part of the cycle the vibratory element and the output element are not in contact and no forces can be transmitted. Although this mechanism is easily described and appears to be rather easy to realize in various ways, there is one major disadvantage; the behavior of the driven member, being out of contact for part of the cycle, is not constantly under control. With a somewhat more complicated kinematic set-up involving two such mechanisms operating $180^{\circ}$ out of phase with each other, this difficulty can be eliminated.

In Fig. 4 we show a smooth non-holonomic realization of our basic $(x, y, z)$-equation introduced above. It consists of a torsional shaft that has a thin disk attached and oscillates with two modes. In this case the disk rolls without slipping on a movable output member $\mathrm{A}$, which slides to produce the output motion. The orientation of the disk changes as it rolls over the output member in such a way that it slants in one direction as it moves back and to the left and in the opposite direction as it moves forward and to the right. Even though there is some coupling between the torsional mode 


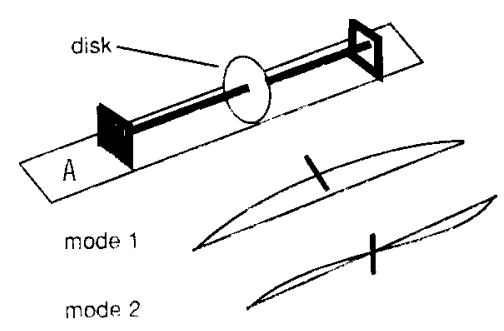

Fig. 4. The twisting rod kinematic realization.

and the first and second transverse modes, there are still only two modes of vibration.

\section{Dynamical Version}

The formula that gives the velocity of the rectilinear motion in terms of the area of the closed curve defined by $x(\cdot)$ and $y(\cdot)$ makes it clear that insofar as purely kinematical solutions are concerned, rectification necessarily involves two degrees of freedom with an independently controllable phase angle between them. The control of the phase angle is necessary to control the speed and direction of the output.

There are also single-driver versions of these mechanisms that stand in relationship to the twodriver situation as 'split phase' electrical motors stand to multiphase electrical motors. Their realization depends on the use of phase shifting to generate a $(u, v)$-pair from, say, $u$ alone. In this case the model has only one input

$\dot{x}=f(x)+g(x) u$

In order to make the system reversible in the sense that the sign of the secular term can be controlled, it is necessary to rely on effects related to a change in the frequency of $u$. The idea is well explained by recasting the model in second-order form. Consider

$\tilde{x}+\epsilon \dot{x}+x=u(t)$

$\dot{z}=x \cdot u$

If $u(t)=\sin \omega t$ and $\epsilon$ is positive but small, then for $w<1, x$ is approximately given by

$x(t) \approx\left(1-w^{2}\right)^{-1} \sin \omega t$

and $\dot{z}$ is approximately the average value of $\left(\sin ^{2} t\right) /\left(1-w^{2}\right)^{-1}$. If $w$ is greater than one, then the same formula applies, except that there is a minus sign because $x$ and $u$ are then nearly $180^{\circ}$ out of phase. Thus in this model the direction of the output motion is controlled by shifting the frequency from one side of the resonance frequency to the other. In this case the speed can only be controlled by controlling the amplitude of $u$.

\section{Small-amplitude Gain}

It is to be observed that unlike the situation one finds in treating conventional systems, the mechanical advantage cannot be evaluated by computing the Jacobian of some map as in the law of levers. In fact, as the amplitude of the driving oscillation goes to zero, the mechanical advantage goes to infinity, reflecting the quadratic nature of the kinematic coupling.

\section{Acknowledgements}

This work was supported in part by the U.S. Department of the Air Force under grant AFOSR-96-00197, in part by the U.S. Army Research Office under grant DAAL03-86-K-0171, and in part by the National Science Foundation under grant CDR-85-00108.

Dr Jair Koiller of the Laboratorio Nacional de Computacio Cientifica, in Rio de Janeiro, has kindly drawn my attention to ref. 9 , which is very much in the spirit of this study.

\section{References}

1 R. S. Muller, From ICs to microstructures: materials and technologies, Proc. IEEE Micro Robots and Teleoperators Workshop, Hyannis, MA, U.S.A., Nov. 1987.

2 J. H. Lang, M. F. Schlect and R. T. Howe, Electric micromotors: electromechanical characteristics, Proc IEEE Micro Robots and Teleoperators Workshop, Hyannis, MA, U.S.A., Nov. 1987.

$3 \mathrm{H}$. Fujita and A. Omodaka, Electrostatic actuators for micromechatronics, Proc IEEE Micro Robots and Teleoperators Workshop, Hyannis, MA, U.S.A., Non. 1987.

$4 \mathrm{R}$. W. Brockett, On the control of vibratory actuators. Proc. 1987 IEEE Conf. Decision and Control, Los Angeles, CA, U.S.A., Dec. 1987, pp. 1418-1422.

$5 \mathrm{Y}$. Ise, Traveling wave ultrasonic motors offer high concession efficiency, J. Elect. Eng., (June) (1986) 66-70.

6 E. T. Whittaker, A Treatise on the Analytical Dynamics of Particles and Rigid Bodies, Cambridge University Press, Cambridge, U.K., 1904.

7 Jack K. Hale, Oscillations in Nonlinear Systems, McGrawHill, New York, 1963.

8 R. W. Brockett, Control theory and singular Riemannian geometry, in P. J. Hilton and G. S. Young (eds.), New Directions in Applied Mathematics, Springer, New York, 1981, pp. 11-27.

9 I. I. Blekhman, The development of the concept of direct separation of motion in nonlinear mechanics, in A. Ya. Ishlinsky (ed.), Theoretical and Applied Mechanics, MIR, Moscow, 1981.

10 R. W. Brockett, Smooth multimode control systems, in L. Hunt and C. Martin (eds.), Proc. 1983 Berkeley-Ames Conference on Nonlinear Problems in Control and Fluid Dynamics, Math Sci Press, Brookline, MA, 1984, pp. $103-$ 110. 


\section{Biography}

Roger W. Brockett received the Ph.D. degree from Case Western Reserve University, Cleveland, $\mathrm{OH}$.

From 1963 to 1969 he taught in the Department of Electrical Engineering at the Massachusetts Institute of Technology, Cambridge. Since then, he has been at the Division of Applied Sciences,
Harvard University, Cambridge, MA where he is An Wang professor of electrical engineering and computer science. His current work is in robotics and computer vision.

Dr Brockett is recipient of the American Automatic Control Council's Donald P. Eckman Award and Richard E. Bellman Award. In 1975 he held a Guggenheim Fellowship for study in mathematical system theory. 